\title{
Assembly Dynamics of FtsZ and ARC6 Plastid-Dividing Proteins
}

\author{
Stanislav Vitha*, Andreas Holzenburg****, Katherine W. Osteryoung***
}

Texas A \& M University, Microscopy \& Imaging Center (MS 2257), **Dept. Biology and Dept. Biochem. \& Biophysics College Station, TX 77843-2257; ***Michigan State University, Dept. Plant Biol., East Lansing, MI 48824.

Chloroplasts and all plastids must divide in order to maintain their numbers in meristematic cells. Plastid division is partially derived from the cell division mechanism of their cyanobacterial endosymbiotic ancestors. In bacteria, the cytoskeletal tubulin-like protein FtsZ assembles into a contractile ring (Z-ring) underneath the plasma membrane at the onset of plastid division and functions as a scaffold for assembly of downstream components of the division machinery. Homologues of FtsZ play a similar role in division of chloroplasts in plants and algae [1,2]. Unlike bacteria, which possess a single FtsZ gene, plants contain two distinct FtsZ families, both of which are essential for plastid division [3]. Studies in E. coli and B. subtilis revealed that the Z-ring is a dynamic structure with rapid exchange of subunits, with half-time of fluorescence recovery after photobleaching (FRAP) about 7 seconds. Moreover, the majority of FtsZ was found to be in the soluble, cytosolic pool, and only $\sim 30 \%$ assembled to a Z-ring [4]. Nothing is known about the FtsZ assembly dynamics in plants, but our previous study suggested that the Z-ring in chloroplasts may be a less dynamic structure [5]. In this study we quantified the relative distribution of the soluble and assembled FtsZ in plastids of Arabidopsis thaliana carrying a fusion of AtFtsZ1-1 with the Green Fluorescent Protein (GFP). We also investigated the ring dynamics of the inner-envelope membraneanchored ARC6, a J-domain protein believed to interact with FtsZ and to stabilize the Z-ring and/or anchor it to the membrane [6].

Arabidopsis plants of wild-type background expressing the AtFtsZ1-1-GFP construct [5] and plants of the arc6 mutant background in which plastid division was restored by the ARC6-GFP fusion construct [6] were germinated on filter paper for one day in continuous white light and then grown in the dark for additional three days in order to minimize chlorophyll synthesis and the associated high autofluorescence of chloroplasts. Seedlings were mounted in water and viewed on a Zeiss Axiophot microscope equipped with a 63x oil immersion objective, a GFP filter set (ex. 450-490 nm, emission 500-550nm) and a Coolsnap cf (Photometrics, Tucson, AZ) CCD camera. Fluorescence intensity was quantified with ImageJ (http://rsb.info.nih.gov/ij). FRAP experiments were performed on a Leica AOBS SP2 confocal microscope. A diffraction-limited spot on the FtsZ or ARC6 ring was photobleached to $20-50 \%$ of the original intensity and the recovery of fluorescence monitored in time-lapse images.

Both AtFtsZ1-1-GFP and ARC6-GFP localized to a ring at mid-plastid (arrowheads in Fig. 1A and B). Slight overexpression of the fusion protein lead to a moderate inhibition of plastid division and the formation of multiple filaments (arrows in Fig. 1) especially in the epidermis of AtFtsZ1-1-GFP transgenic plants. These and other data (not shown) suggest that the fusion proteins are functional. The quantification of fluorescence and FRAP data collection is under way. These experiments will be crucial in determining the dynamism and the equilibrium state of FtsZ ring assembly and in further defining the functional role of the ARC6 protein. 



Figure 1. GFP Fluorescence localization of AtFtsZ1-1 and ARC6 fusion proteins in chloroplasts of Arabidopsis thaliana. A. AtFtsZ1-1-GFP. B. ARC6-GFP. Bar $=10$ $\mu \mathrm{m}$.

\section{References:}

1. K.W. Osteryoung and R.S. McAndrew, Annu. Rev. Plant Physiol. Plant Mol. Biol. 52 (2001) 315.

2. H. Kuroiwa, et al., Planta 215 (2002) 185.

3. K.W. Osteryoung, et al., Plant Cell 10 (1998) 1991.

4. D. Anderson, et al., J. Bacteriol. 186 (2004) 5775.

5. $\quad$ S. Vitha, et al., J. Cell Biol. 153 (2001) 111.

6. S. Vitha, et al., Plant Cell 15 (2003) 1918. 